Research Paper

\section{A Self-governing Fourth-order Nonlinear Diffusion Filter for Image Noise Removal}

\author{
Mohammad Reza Hajiaboli ${ }^{\dagger 1}$
}

Fourth-order nonlinear diffusion denoising filters are providing a good combination of the noise smoothing and the edge preservation without creating the staircase artifacts on the filtered image. However, finding an optimal choice of model parameters (i.e. the threshold value in a diffusivity function and a time step-size for stability of the numerical solver) is a challenging problem and generally, these model parameters are image-content dependent. In this paper, a fourth-order diffusion filter is proposed in which the diffusivity function is a function of modulus of the gradient of the image. It is shown that this setting for the diffusivity function can lead to a robust and fast convergent filter in which the model parameters are reduced to the only threshold value in the diffusivity function that can be estimated. A data-independent time step-size has been analytically found to guarantee the convergence of numerical solver of the proposed filter. Although this time step-size is smaller than the ones typically used, it is typically used, it is shown that the numerical solver of the proposed filter can provide a significantly fast convergence rate compared to the classical filter due to an improvement of the image selective smoothing obtained by the diffusivity function of the proposed filter. Simulation results demonstrate that the quality of denoised images obtained by the proposed filter are noticeably higher than the ones from the existing filters.

\section{Introduction}

In the last two decades, the use of partial differential equations (PDEs)-based filters in image processing has significantly increased. This paper mainly focuses on the class of PDE-based filter known as nonlinear/anisotropic diffusion filters. The first kind of these filters is introduced by Perona and Malik ${ }^{1)}$ in 1990 in which the denoised image is the solution of a nonlinear second-order PDE. Since then, a great deal of research in this field has led to the introduction of a variety $\dagger 1$ Faculty of Engineering and Computer Science, Department of Electrical Engineering
Concordia University, Montreal, Canada of nonlinear diffusion filters (see Refs. 2) and 3) as a few examples). Although the method proposed by Perona and Malik and its variants are known as good edge preservation denoising filters, they tend to produce blocky effects in the images ${ }^{4)}$, since only a piecewise constant image can be the solution of the associated PDE.

An effective solution to this problem has been introduced by You and Kaveh ${ }^{4)}$ in which a fourth-order PDE is used for image noise removal. Because this filter supports a planar approximation of the image in the solution of the PDE, a significant improvement in the ramp edge preservation and a dramatic reduction of blocky effects are achieved by using this fourth-order diffusion scheme. However, from practical stand view, using the fourth-order nonlinear diffusion filter for noise removal comes with the following difficulties:

(1) Finding the optimal choice for model parameters (i.e., the threshold value in the diffusivity function and the time step-size in the numerical solver of this filter) is very difficult and they are mainly image-content dependent. Thus, fourth-order filters including the recently developed ones ${ }^{5)-7 \text { ) }}$ are mainly known as manual or man-operated noise removal techniques.

(2) The optimal choice of parameters are usually led to a very slow convergence rate for these filters as it has been reported in Refs. 4) and 5).

In fact, the Fourth-order diffusion filter dampens high spatial frequency components (i.e. noise and step edges) much faster than the second-order diffusion ${ }^{5)}$. This feature can potentially result in the edge distortion during the evolutionary process of the image denoising especially when the smoothing strength of the filter for the detected edges is not effectively reduced by a diffusivity function. The diffusivity function of the fourth-order diffusion filters is a function of the absolute value of Laplacian of the evolved image and it is more sensitive to the noise compared to the diffusivity function of the second-order nonlinear diffusion filters, which is a function of the modulus of the gradient of the evolved image. Thus, the well-established techniques for estimation of the model parameters in second-order nonlinear diffusion filters are not directly applicable in the fourthorder filters. On the other hand, due to a severe nonlinearity of the fourth-order dynamic flow, finding the optimal time step-size in the numerical solver of these filters is very difficult and it is usually obtained after a long tail of try and error.

In this paper, a self-governing fourth-order nonlinear diffusion filter is proposed 
95 A Self-governing Fourth-order Nonlinear Diffusion Filter

in which the model parameters are reduced to only one parameter (i.e., the threshold value in the diffusivity function) that can be estimated. The diffusivity function of the proposed filter is a function of the modulus of the gradient of the evolved image, thus it provides the possibility of adoption of a well-established estimation mechanism for the threshold value after a necessary adjustment. Due to a good control of the strength of the diffusion of the filter on edges and smooth regions of the image, it is shown that the time step-size in the numerical solver can be set to a data-independent one, which is smaller than the optimal one and yet the proposed filter provides a fast convergence rate comparing to that of the classical filter.

This paper is organized as the following: In Section 2, a brief review of the nonlinear diffusion filters and their transition from second-order to fourth-order is described. In the next section, the proposed filter is presented through subsections. First, the associated PDE of the proposed filter is introduced and it has been shown that the filter still can support the planar approximation of the image. In the next subsection, the estimation of the threshold value in the diffusivity is discussed and finally in the last subsection, the data-independent time step-size for the numerical solver is analytically derived. Section 4 is devoted to comparative results. In this section, the performance of the proposed filter is compared with those of the classical and recently developed filters, where it has been shown that the proposed method can outperform the other fourth-order filters in terms of the quality of the denoised images and the convergence rate of the filters. Finally, Section 5 concludes the paper by highlighting the significance of the proposed filter.

\section{A Brief Review}

The basic diffusion equation of Perona and Malik ${ }^{1)}$ for a two dimensional image intensity function of $u$ in Cartesian coordinate of $x$ and $y$ is given by

$$
\partial u / \partial t=\operatorname{div} .(c(\|\nabla u\|) \nabla u),
$$

where $c($.$) is the diffusivity function by which the diffusion coefficient is cal-$ culated and $t$ is time. Symbols of div. and \|. || are used for the mathematical notations of divergence and Euclidean norm $\left(\|\nabla u\|=\sqrt{u_{x}^{2}+u_{y}^{2}}\right)$ respectively.
The diffusivity function is a positive and non-increasing function of $\|\nabla u\|$. One of these diffusivity functions defined by Perona and Malik is given by

$$
c(|\nabla u|)=k^{2} /\left(k^{2}+\|\nabla u\|^{2}\right),
$$

where $k$ is the so-called contrast parameter. There are some effective automatic mechanisms for estimation of the contrast parameter such as the schemes introduced in Refs. 1) and 3).

You and his colleagues ${ }^{8}$ carried out a detailed analysis to show that the solution of (1) is equal to minimization of an energy functional. If the diffusivity function of (2) is used then the energy functional is

$$
R(u)=\int_{\Omega} \frac{k^{2}}{2} \ln \left(k^{2}+\|\nabla u\|^{2}\right) d x d y
$$

where $\Omega$ is the region of support of $u$. $R(u)$ is minimized when $\|\nabla u\|^{2}$ is minimum, which leads to a piecewise constant approximation of $u$. Therefore, formation of staircase artifacts on the ramp edges is unavoidable. In order to resolve this problem, You et al. ${ }^{4)}$ introduced a fourth-order PDE-based denoising method in which the denoised image is obtained by minimization of the potential function given by

$$
E(u)=\int_{\Omega} f\left(\left|\nabla^{2} u\right|\right) d x d y
$$

where $f^{\prime}(s)=s c(s)$. Using the same diffusivity function of (2), the potential function of $E(u)$ can be written as

$$
E(u)=\int_{\Omega} \frac{k^{2}}{2} \ln \left(k^{2}+\left|\nabla^{2} u\right|\right) d x d y,
$$

meaning that $E(u)$ is minimized when $\left|\nabla^{2} u\right|$ is minimum. Therefore, the ramp regions of $u$ (i.e., the regions where $\left|\nabla^{2} u\right|=0$ ) are fitted in the solution of the associated fourth-order PDE. The solution of the minimization problem of (4) after using Euler equation followed by gradient descent procedure is given by

$$
\partial u / \partial t=-\nabla^{2}\left(c\left(\left|\nabla^{2} u\right|\right) \nabla^{2} u\right)
$$

By the forward Euler approximation of the $\partial u / \partial t$, the numerical solver of $(6)$ is given by 


$$
\begin{aligned}
u^{n+1} & =u^{n}-d t \times \nabla^{2}\left(c\left(\left|\nabla^{2} u^{n}\right|\right) \nabla^{2} u^{n}\right), \\
u^{0} & =u_{0} \quad \text { and } \quad n=0,1, \cdots, N
\end{aligned}
$$

where $n$ is the number of iterations, $d t$ is a time step-size and $u_{0}$ is a noisy image used as an initial condition. This process is an iterative process. In order to protect the edges from over-smoothing, the process needs to be stopped at a certain number of iterations denoted by $N$.

Apart from a significant advancement in reduction of blocky effects on the denoised image using Eq. (6), the optimal parameter setting for its numerical solver in Eq. (7) leads to a very slow convergence rate especially, when the level of contaminating noise is moderately high. A recently developed technique known as a hybrid model of fourth-order $\mathrm{PDE}^{6}$ ) addresses this problem and by using a relaxed median filter ${ }^{9)}$ tends to improve the quality of the denoised image, when the image is highly noisy. The numerical model of this filter is given by

$$
u^{n+1}=R M_{\alpha \omega}\left(u^{n}-d t \times \nabla^{2}\left(c\left(\left|\nabla^{2} u^{n}\right|\right) \nabla^{2} u^{n}\right)\right),
$$

where RM denotes the relaxed median filter with a lower bound of $\alpha$ and upper bound of $\omega$. This filtering process needs a lower number of iterations to give an estimation of the denoised image. However, the quality of the denoised image by Eq. (8) is dramatically low, as it is shown in Section 4.

The important point is that both of these techniques are still suffering from a lack of model parameter estimation mechanisms particularly for the contrast parameter, $k$, and the time step-size, $d t$. As mentioned earlier, the performance of these filters strongly depends on the selection of these parameters and unfortunately, the optimal parameters selection is image-content-dependent.

Besides these nonlinear diffusion filters, another class of PDE-based filters known as regularization techniques has been widely used for image restoration. The classical paper of Rudin, Osher and Fatemi ${ }^{10}$ ) introduces a nonlinear filter of this kind in which the PDE to be solved is of the second-order. Therefore, the same problem of formation of staircases on the ramp regions of the image motivates the researchers to introduce the new regularization techniques by solving the higher order PDE such as the ones in Refs. 11) and 12).

However, the focus of this paper is on the fourth-order diffusion based tech- niques as they have been reviewed earlier. In the following section, a new filtering scheme is introduced in which the challenge of model parameter settings is addressed and a robust solution to this problem is given.

\section{The Proposed Filter}

\subsection{The Associated PDE}

The ability of edge preservation in the fourth order PDE-based denoising method strongly depends on the extent by which the diffusivity function, $c($.$) ,$ can detect the edges and reduce the strength of the smoothness of the filter for these detected edges. However, when the diffusivity function is a function of the absolute value of the Laplacian of the image, the probability of a false edgedetection by the diffusivity function is significantly higher comparing to the case that the diffusivity function is a function of the modulus of the gradient. In fact, as much as the order of the derivative of the image is higher, the sensitivity to the noise is higher ${ }^{13)}$. Therefore, the new PDE for denoising is introduced in the form of

$$
\partial u / \partial t=-\nabla^{2}\left(c(\|\nabla u\|) \nabla^{2} u\right),
$$

in which the diffusivity function is a function of the gradient modulus of the evolved image (unlike Eq. (6) where the diffusivity is a function of $\left|\nabla^{2} u\right|$ ). Similar to Eq. (7), the numerical solver of Eq. (9) is given by

$$
\begin{aligned}
u^{n+1} & =u^{n}-d t \times \nabla^{2}\left(c\left(\left\|\nabla u^{n}\right\|\right) \nabla^{2} u^{n}\right), \\
u^{0} & =u_{0} \quad \text { and } \quad n=0,1, \cdots, N .
\end{aligned}
$$

As the results in the next section show, this simple change can lead to a substantial improvement in the quality of the denoised image and the convergence rate of the filter. The most important feature that needs to be guaranteed by the proposed filter is its ability to support the planar approximation of the evolved image. Unlike the You and Kaveh filter that has a closed form potential function of Eq. (5) by which the planar approximation of the $u$ could be shown, for the proposed filter this feature can be shown by looking at its associated PDE. In fact, solution of the Eq. (9) or Eq. (6) in the steady state means that $\partial u / \partial t \rightarrow 0$. For a ramp region of the image, $\partial u / \partial t=0$, because $\nabla^{2} u=0$. Therefore, the 
97 A Self-governing Fourth-order Nonlinear Diffusion Filter
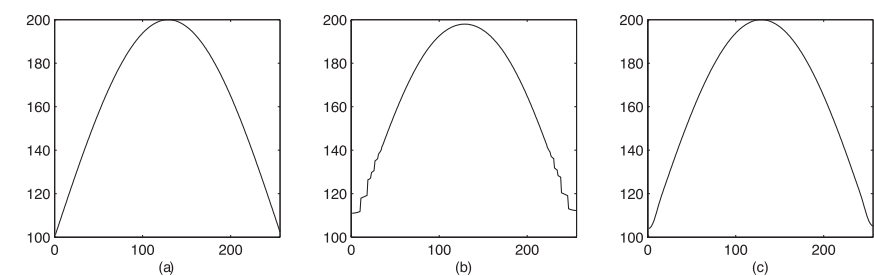

Fig. 1 Comparing the result of the proposed filter with the result obtained by Perona Malik filter in term of formation of artifacts. Fig. 1-(a) is the original image, Fig. 1-(b) is the result of Perona-Malik filter $(t=150)$ and Fig. 1 -(c) is the result obtained by the proposed filter $(t=150)$.

dynamic flow of the Eqs. (9) and (6) on the ramp region of the image is reaching to the steady state regardless of their diffusivity function and the diffusion coefficient calculated by it. Based on this argument, one can conclude that the planar approximation of the intensity function of $u$ can be supported by the proposed filter. The results demonstrated in Fig. 1 give a comparison between the performance of the proposed filter and Perona and Malik filter, where the signal shown in Fig. 1-(a) is been filtered for $t=150 \mathrm{~s}$. The diffusivity function for the proposed filter and Perona and Malik filter is chosen to be Eq. (2). While the result obtained by Perona and Malik filter shown in Fig. 1-(b) apparently suffers from the formation of the blocky effect, the results of the proposed method in Fig. 1-(c) does not exhibit any sign of the formation of staircase artifacts.

However, the main advantage of the proposed filter compared to the You and Kaveh is in its ability of running in an unsupervised fashion. In the following subsections, this aspect is explored in details.

\subsection{Estimation of The $k$ Parameter}

When the diffusivity function in the proposed method is a function of $\|\nabla u\|$, the optimal threshold value, $k$, in $c($.$) , is proportionally related to the noise level { }^{14)}$ and can be estimated by Canny noise-estimation technique ${ }^{15)}$ as the following:

A histogram of the magnitude values of the gradient throughout the image is computed and $k$ is set to the $\chi \%$ value of its integral at each iteration.

This way of estimation of the $k$ has been suggested by Perona and Malik and followed by many other researchers in the domain of the second-order diffusion filters as a reliable scheme, where the value of $\chi$ is set in the range of $[80,90]$.

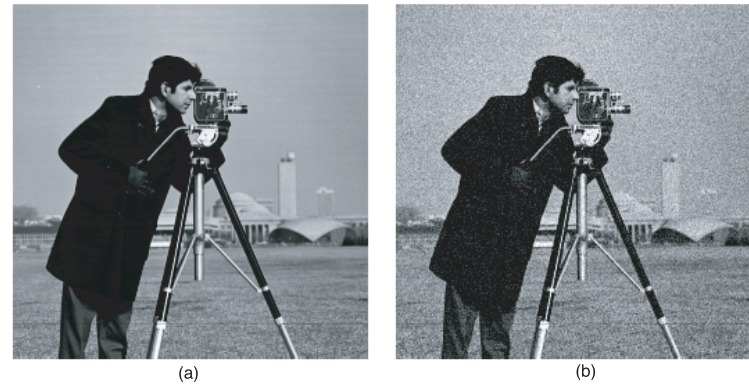

(b)

Fig. 2 The test image of cameraman and its degraded one. The degradation model is additive white Gaussian noise (AWGN) with standard deviation (SD) of 15

The amount of the reduction of the diffusivity on the edges and the strength of the smoothness obtained by the second-order diffusion filter are matched up so that the process of the edge distortion is reduced dramatically before the noise is removed. However, when it is used for the proposed diffusion filter, it will result in a slightly stronger edge smoothening due to the higher diffusion strength of the fourth-order filter compared to the second order filter.

In order to take into account this discrepancy between the diffusion strength of the second and fourth-order filters, the threshold value of the diffusivity function of the proposed filter needs to be reduced to increase the edge preservation capability of the filter. To do so, the $\chi$ in the proposed filter is set to 40 . The rational behind this choice can be explained by uncorrelation criterion ${ }^{17)}$. In fact, if the noise and signal are uncorrelated then, in a fine tuned diffusion filter, the value of the correlation of the residual image of $u_{0}-u(t)$ and the developing image of $u(t)$ with respect to $t$ (i.e., $n \times d t$ in the discrete domain) needs to be a unimodal curve with a single minimum.

Experiments on a variety of test images contaminated with different level of additive white Gaussian noise (AWGN), i.e., an uncorrelated stationary noise, shows that this feature does not hold true unless the value of $\chi$ is reduced and a suitable choice is $\chi=40$. The result of one of these experiments is given in Fig. 3. This result is obtained for a test image of Cameraman contaminated by AWGN with standard deviation (SD) of 15 shown in Fig. 2. The upward spike in the correlation curve for $\chi=80$ and 60 in the early stage of the development 
98 A Self-governing Fourth-order Nonlinear Diffusion Filter

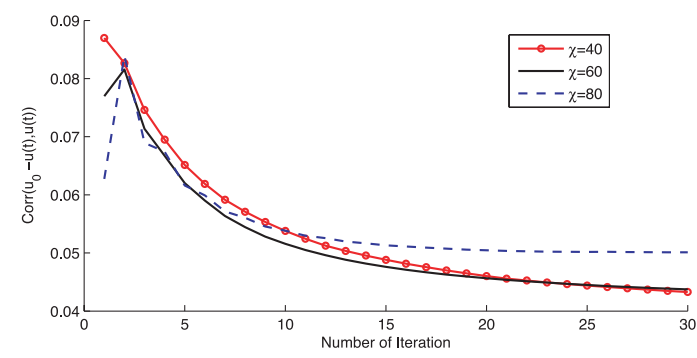

Fig. 3 Comparing the correlation of the residual image of $u_{0}-u(t)$ and $u(t)$ for three different value of $\chi$.

of the denoised image shows that the estimated value of $k$ in these cases is large enough to lead to removal of a part of the structure of the image (i.e. mainly low contrast edges) and their representation in the residual image of $u_{0}-u(t)$ while choosing the $\chi=40$ can prevent the smoothness of these edges.

\subsection{Stability Condition}

The other important aspect of the fourth-order PDE-based denoising techniques is the stability of the numerical solver in order to guarantee the convergence of the solution. Setting a small time step-size, $d t$, in Ref. (10), can guarantee the stability of the dynamic flow, however if $d t$ is too small, it results in numerous number of iterations. Therefore, finding an optimal time step-size is crucial in order to reduce the convergence time. On the other hand, if the diffusivity function can distinguish between the noise and edge with a high degree of certainty, the convergence rate of the PDE-based denoising filter can be dramatically increased. Comparing the diffusion coefficient map of noisy image of "cameraman" given in Fig. 2 at the $30^{\text {th }}$ iteration for the You and Kaveh filter with the one obtained by the proposed filter shows that for the proposed filter, the wider regions of the image can be detected as smooth regions. Therefore, the noise reduction is significantly faster than that of Eq. (7). Thus, finding a constant $d t$ as a data-independent time step-size for Eq. (9) (which is smaller than the optimal time step-size) and setting the numerical solver of Eq. (10) so that it operates with this time step-size, make the proposed filter applicable to a wide category of images without any stability concern and still deliver a good convergence rate.

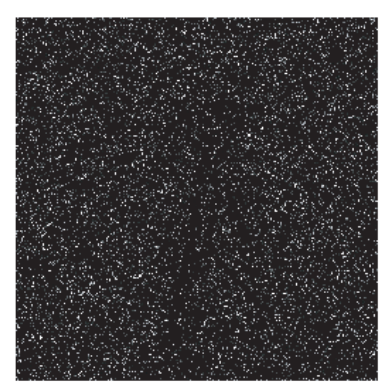

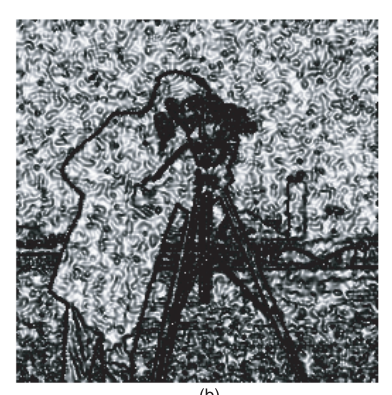

Fig. 4 Comparing the map of $c\left(\left|\nabla^{2} u\right|\right)$ in You and Kaveh filter, (a), with the map of $c(|| \nabla u||)$ in the proposed filter, (b), for the noisy image of cameraman shown in Fig. 2. The bright regions in the both maps indicate the regions in which a strong diffusion is carried out.

To find a time step-size, $d t$, for the numerical solver in Eq. (10) so that it is always convergent to the steady state value, it is needed to show that $\partial u / \partial t \rightarrow 0$ when $t \rightarrow \infty$. If $c(\|\nabla u\|) \approx 0$ the changes in $u$ is almost zero and $\partial u / \partial t \approx 0$. Therefore, the worst condition for stability is when $c(\|\nabla u\|)=1$. Note that $c(\|\nabla u\|)$ is bounded in $(0,1]$. Therefore, under this worst condition for stability when $c(\|\nabla u\|)=1$, the numerical solver can be written in the form of

$$
u^{n+1}=u^{n}-d t \times\left((L * L) * u^{n}\right),
$$

where $L$ is a small Laplacian kernel and symbol of $*$ denotes the convolution operation. If $u$ is arranged in the column-wise order, the linear system in (11) can be written as a system of state equations given by

$$
u^{n+1}=(I+S) \times u^{n}
$$

where $(I+S)$ is the state matrix of Eq. (11) in which $S$ is an sparse matrix representing the convolution of $u$ with the small convolution kernel of $-d t \times$ $(L * L)$ and $I$ is the identity matrix. When $L$ is a standard Laplacian kernel given by

$$
L=\left[\begin{array}{ccc}
0 & 1 & 0 \\
1 & -4 & 1 \\
0 & 1 & 0
\end{array}\right]
$$

then $-d t \times(L * L)$ is 


$$
-d t \times(L * L)=d t \times\left[\begin{array}{ccccc}
0 & 0 & -1 & 0 & 0 \\
0 & -2 & 8 & -2 & 0 \\
-1 & 8 & -20 & 8 & -1 \\
0 & -2 & 8 & -2 & 0 \\
0 & 0 & -1 & 0 & 0
\end{array}\right]
$$

which means that $S$ is a sparse matrix that the nonzero matrix values in each row are in the following order:

$$
d t \times\left[\begin{array}{lllllllllllll}
-1 & -2 & 8 & -2 & -1 & 8 & -20 & 8 & -1 & -2 & 8 & -2 & -1
\end{array}\right](15)
$$

while -20 is always in the main diagonal of $S$.

For stability of the state system in Eq. (12), $d t$ should be set so that the spectral radius, $(S P)$, of $(I+S)$ is less than one. On the other hand, the same value of $d t$ leads to the stability of

$$
u^{n+1}=-(I+S) \times u^{n},
$$

However, the state system in Eq. (16) can be written in standard form of Jacobi solver in the form of

$$
u^{n+1}+((2 I+S)-I) u^{n}=0 .
$$

Stability of the Jacobi solver in Eq. (17) can be obtained if matrix $(2 I-S)$ is strictly row-wise diagonally dominant (see Ref.16) page 626 for proof). By definition, the matrix $A_{i j}$ is strictly row-wise diagonally dominant if

$$
\left|a_{r r}\right|>\sum_{j \neq r}\left|a_{r j}\right| \text { for } r=1,2, \cdots, n .
$$

This condition for $(2 I-S)$ means that if $2-20 d t>44 d t$ is satisfied the diffusion Eq. (17) and consequently Eq. (12) are convergent. Recalling the fact that the diffusion equation of Eq. (12) is obtained under the maximum diffusion strength of the proposed filter in Eq. (10), it means that when the time step-size is $d t<$ 0.0313 , the proposed filter using $L$ as a discrete approximation of Laplacian is data-independently stable.

\begin{tabular}{|c|c|c|c|c|c|c|}
\hline \multicolumn{3}{|c|}{ Noisy Image $\mathrm{SD}=15$} & \multicolumn{4}{|c|}{ Denoised Image } \\
\hline Images & SNR $(\mathrm{dB})$ & Method & SNR (dB) & FOM & $\mathrm{N}$ & CPU/Iter \\
\hline \multirow{3}{*}{ Pepper } & \multirow{3}{*}{10.96} & Proposed & 17.67 & 0.9108 & 90 & 0.08 \\
\hline & & (7) & 15.75 & 0.8710 & 3047 & 0.03 \\
\hline & & (8) & 15.10 & 0.7996 & 2 & 0.15 \\
\hline \multirow{3}{*}{ Cameraman } & \multirow{3}{*}{12.39} & Proposed & 17.28 & 0.9465 & 39 & 0.08 \\
\hline & & (7) & 16.60 & 0.9453 & 3115 & 0.03 \\
\hline & & $(8)$ & 13.66 & 0.7345 & 1 & 0.16 \\
\hline \multirow{3}{*}{ House } & \multirow{3}{*}{9.74} & Proposed & 17.48 & 0.8413 & 270 & 0.08 \\
\hline & & (7) & 15.82 & 0.8252 & 3904 & 0.03 \\
\hline & & (8) & 15.34 & 0.7468 & 2 & 0.16 \\
\hline \multicolumn{4}{|c|}{ Noisy Image $\mathrm{SD}=25$} & \multicolumn{3}{|c|}{ Denoised Image } \\
\hline \multirow{3}{*}{ Pepper } & \multirow{3}{*}{6.55} & Proposed & 15.19 & 0.8435 & 210 & 0.08 \\
\hline & & (7) & 12.91 & 0.8102 & 10550 & 0.03 \\
\hline & & (8) & 13.19 & 0.7602 & 3 & 0.15 \\
\hline \multirow{3}{*}{ Cameraman } & \multirow{3}{*}{7.98} & Proposed & 14.93 & 0.8794 & 210 & 0.08 \\
\hline & & (7) & 13.76 & 0.9044 & 11530 & 0.03 \\
\hline & & $(8)$ & 12.21 & 0.6798 & 2 & 0.16 \\
\hline \multirow{3}{*}{ House } & \multirow{3}{*}{5.32} & & 15.28 & 0.7919 & 490 & 0.08 \\
\hline & & (7) & 13.55 & 0.8012 & 13940 & 0.03 \\
\hline & & (8) & 13.22 & 0.7174 & 6 & 0.16 \\
\hline
\end{tabular}

\section{Comparative Results}

In this section, we are presenting the comparative results of the proposed filter with two other Fourth-order filters. These filters are as the following:
Table 1 Objective comparison results: Degradation model is AWGN.

(1) The proposed filter with $d t=0.031$ and contrast parameter, $\mathrm{k}$, estimated by the histogram-based mechanism explained in Section 3.2.

(2) The filter of (7) introduced by You and Kaveh ${ }^{4)}$ with the suggested parameters setting of $d t=0.25$ and $k=1$.

(3) The filter of (8) introduced in ${ }^{6)}$ with the suggested parameters setting of $d t=0.1, k=3, \alpha=3$ and $\omega=5$.

Three test images of "Pepper", "Cameraman" and "House" have been corrupted by AWGN with $\mathrm{SD}=15$ and 25. In Table 1, an objective comparison between the performances of these filters is presented. This comparison is in terms of quality of the denoised image and computational cost of the filters. For quantifying the quality of the denoised image, signal-to-noise ratio (SNR) and Pratt's figure of merit (FOM) ${ }^{18)}$ of the denoised image are calculated. FOM is an objective quantitative performance measure has often been used in the edge detection evaluation given by 


$$
F O M=\frac{1}{\max \left(u_{D}, u_{I}\right)} \sum_{i=1}^{u_{D}} \frac{1}{1+\beta d_{i}^{2}}
$$

where $u_{D}$ is the number of detected edge points, $u_{I}$ is the number of ideal edge points (ground truth), $\beta$ is a positive scaling factor (often chosen to be $1 / 9$ and so that here), and $d_{i}$ is the edge deviation or error distance for the $i^{t h}$ detected edge pixel. The edge detector for ground truth and denoised image is chosen to be the Sobel edge detector as a built-in function in Matlab. The value of FOM is bounded in range of $(0,1]$ and as it is closer to one it shows a better ability of the filter to preserve the edges.

The results show that the proposed filter constantly produces the denoised image with a significantly higher SNR. The calculated FOM for the denoised images shows that the proposed method is able to preserve the edges in the denoised image as good as the classical filter of Eq. (7) and much better than the fast convergent filter of Eq. (8). It is important to note that the results are obtained at the optimal number of iterations in which the maximum SNR during the evolutionary process of the filters are achieved. If the iterative filtering process is continued after the optimal number of iterations, the SNR of the denoised image is reduced due to the over-smoothness of the edges.

The other important feature in the proposed filter is its fast convergence rate. From practical stand view, the convergence time is measured as an overall computational time of the filter to reach to the highest SNR. The computational burden of the filters is measured as CPU time of each iterations (CPU/Itre.) multiply by the number of the iterations $(\mathrm{N})$ provided that they are filtering the same image on the same computer (the computer used for these simulations was P4 $3 \mathrm{GHz}$ with $1 \mathrm{~GB}$ DRAM at $533 \mathrm{MHz}$ ). From this perspective, the convergence time of Eq. (8) is smaller than the one obtained by the proposed filter and Eq. (7), however the maximum SNR is significantly lower than that of the other filters and the measured FOM for the denoised image by Eq. (8) indicates that this fast convergence comes with the strong over-smoothing of the edges. On the other hand, the good edge preservation obtained by Eq. (7) leads to a very slow convergence rate of this filter. However, the proposed filter stand at the middle of these two extreme cases. It means that the convergence time of the proposed
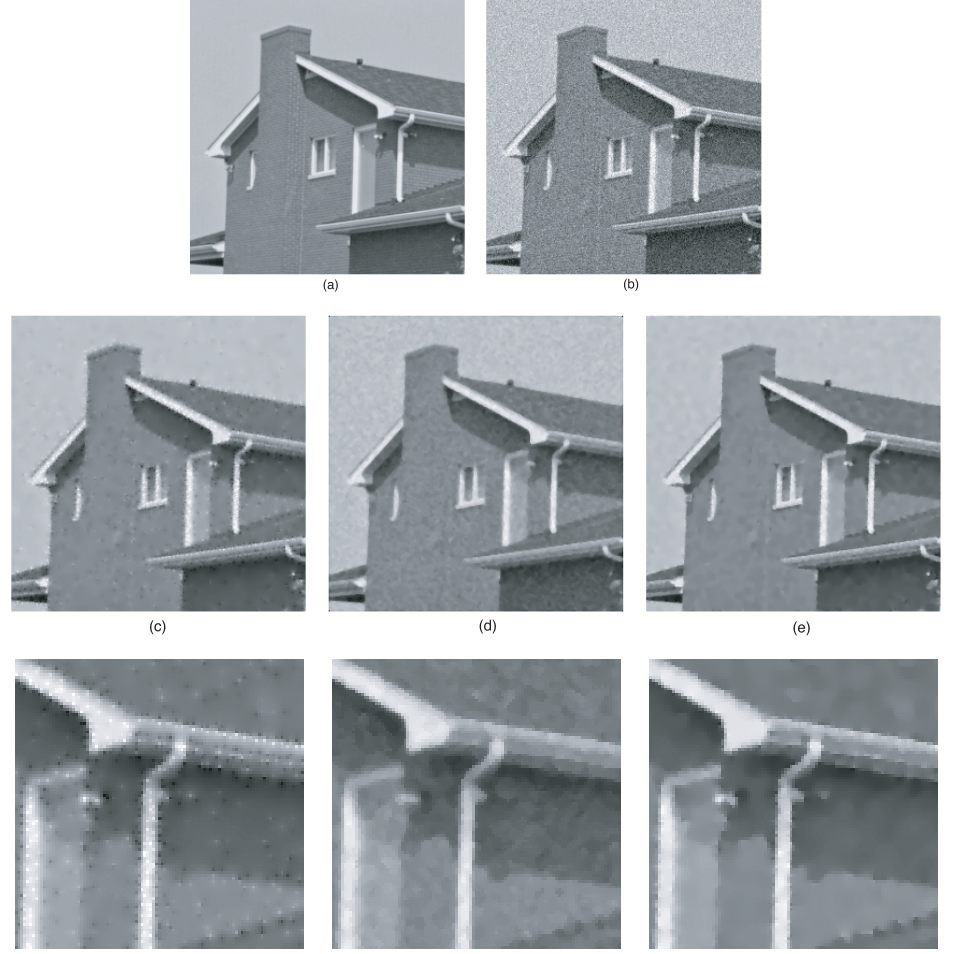

Fig. 5 Comparing the perceptual quality of the results. The images labeled (a) to (e) are as the following: (a) noiseless image, (b) noisy image (AWGN with $\mathrm{SD}=15$ ), (c) denoised image using (6), (d) denoised image using (8) and (e) the result obtained by proposed filter. A magnified region of each denoised image is shown in the third row.

filter is higher than Eq. (8) and remarkably lower than Eq. (7) while both SNR and FOM are almost higher than or comparable to those of the others.

In Figs. 5 and $\mathbf{6}$, the perceptual quality of the denoised image by the proposed filter is compared with the ones obtained by the other filters. In the first two rows, the whole image and in the third row, a magnified portion of the denoised image are shown. The images are labeled from (a)-(e). The first two images (a) and (b) are the noiseless and the noisy images. In Fig. 5, the test image House and its noisy version degraded by AWGN with $\mathrm{SD}=15$ are shown in sections 

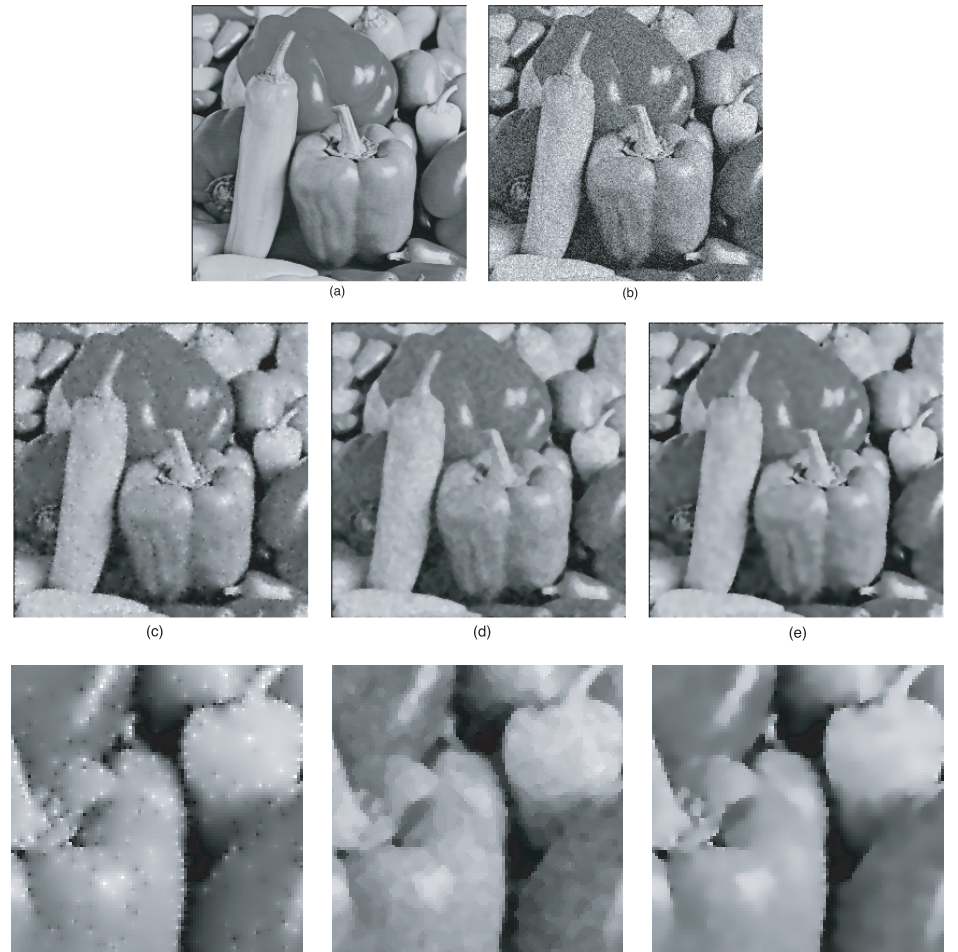

Fig. 6 Comparing the perceptual quality of the results. The images labeled (a) to (e) are as the following: (a) noiseless image, (b) noisy image (AWGN with $\mathrm{SD}=25$ ), (c) denoised image using (6), (d) denoised image using (8) and (e) the result obtained by proposed filter. A magnified region of each denoised image is shown in the third row.

(a) and (b) and sections (c) to (e) are the results of the filters (7), (8) and the proposed filter respectively. In Fig. 6, the results for the test image of Pepper degraded by AWGN with $\mathrm{SD}=25$ are shown with the same order described above. On the surface of the denoised image by Eq. (7) some speckle noise are formed. This drawback is known and addressed by You and Kaveh ${ }^{4)}$ and it is as a result of choosing a small value for $k$ in the diffusivity function, however this setting is necessary to protect the edges from over-smoothing. In the section (d) of the Fig. 5 and 6 , the denoised image by Eq. (8) are shown where one can clearly see that this filter in spite of its fast convergence rate does not provide a good edge preservation and moreover the dominance of the effect of the relaxed median filter used in this filtering scheme leads to the formation of staircase artifacts on the smooth regions. This major drawback is clearly visible in magnified image of Fig. $6(\mathrm{~d})$. The last image, shown in Fig. 5 and $6(\mathrm{e})$ are the result of the proposed filter in which the extent of the denoising and edge preservation is noticeably better than those of the other filters.

This improvement in the results obtained by the proposed method is largely because of the modification of the diffusivity function. As it is shown in Fig. $\mathbf{4}$, choosing the diffusivity function as a function of the first-order derivative of the evolved image in the proposed filter leads to a better edge detection compared to the diffusivity functions of the other fourth order filters in which the diffusivity is a function of the second-order derivative of $u$. Thus, one can expect that noise is more effectively reduced with much less over-smoothness of the edges.

However, the effect of the setting parameters of $d t$ and $k$ in the enhancement of the results of the proposed filter are not negligible. In the following, these effects are described in more details:

(1) The effect of $\boldsymbol{d t}$ : The choice of $d t$ has the effect on the accuracy of the numerical solver so that if $d t$ is decreased the numerical solver can approximate the result with higher accuracy, however the convergence time is also increased. Thus, setting the time step-size, $d t$, should satisfy not only stability of the numerical solver but also it should provide a good trade-off between the accuracy and convergence time. One of the advantages of the proposed filter is that these requirements have been achieved with a very small $d t$. Choosing the same $d t$ for other filters will not hinder the stability of those, however it is not practically an optimal choice. Reduction of the time-step size for the filter of Eq. (7) leads to the increase of the convergence time, which is already very big and for filter of Eq. (8), $d t$ is already much smaller than that of filter Eq. (7) and it does not provide any better results. In fact, the significant edge distortion in the result of this filter (the lowest FOM) is due to apply of a relax median filter in each iteration and any farther reduction of $d t$ will not improve the edge preservation capability of the filter.

(2) The effect of $\boldsymbol{k}$ : The balance between the edge preservation and noise 
102 A Self-governing Fourth-order Nonlinear Diffusion Filter

reduction ability of these diffusion based filters is determined by the threshold value of $k$. It means the reduction of this value may increase FOM of the results but result in forming of speckle noise on the surface of the image specially for filter (7) and it reduces the SNR of the results. Thus, the value of $k$ for filters of Eqs. (7) and (8) is set manually to provide a good balance between the SNR and FOM of the results. In this numerical study, these value are chosen based on the suggestion of the authors of the original papers in Refs. 4) and 6). In the proposed filter, an estimation mechanism relates the optimal value of $k$ to the noise level. By reduction of the noise level during the development of the denoised image, $k$ is gradually reduced to increase the edge preservation capability of the filter and since it is along with reduction of the noise, the speckle noise is not formed on the denoised image.

Finally, the result of the proposed method is compared with that of the PeronaMalik for two dimensional image of Cameraman (shown in Fig. 2). As the comparative results on one dimensional signal in Fig. 1 shows, the second order filter of Perona-Malik creates the staircase artifacts. This drawback in a two dimensional image leads to unnatural (cartoon-like) looking of the processed image. The results in Fig. 7 obtained after filtering the noisy image of Cameraman shown in Fig. 2-(b) by Perona-Malik and proposed filters. The model parameter of $d t$ for both filters is set to $d t=0.031$ and the same histogram-based estimation mechanism described in Section 3.2 is used for the estimation of $k$, however $\chi$ for Perona-Malik is chosen to be 80 as it is suggested in 1). The total number of iteration for both filters is set to 150. Comparing the result of Perona-Malik (shown in Fig. 7 (a)) with that of the proposed filter in Fig. 7-(b) demonstrates the advantage of using the fourth order filter to preserve the natural perception of the processed image and prevent the formation of the staircase artifacts.

However, SNR of the results of the proposed filter is not as good as the one obtained by Perona-Malik (SNR of denoised image by Perona-Malik and the proposed filter are $17.43(\mathrm{~dB})$ and $17.10(\mathrm{~dB})$ respectively). This shortcoming is due to the fact that a part of the noise mainly at piecewise constant regions of the image (e.g., the background region) can not be completely removed by the fourth-order filters because before $\|\nabla u\|$ becomes zero (i.e the best approximation for these regions and it is the solution of the Perona-Malik filter) the $\left|\nabla^{2} u\right|$ can
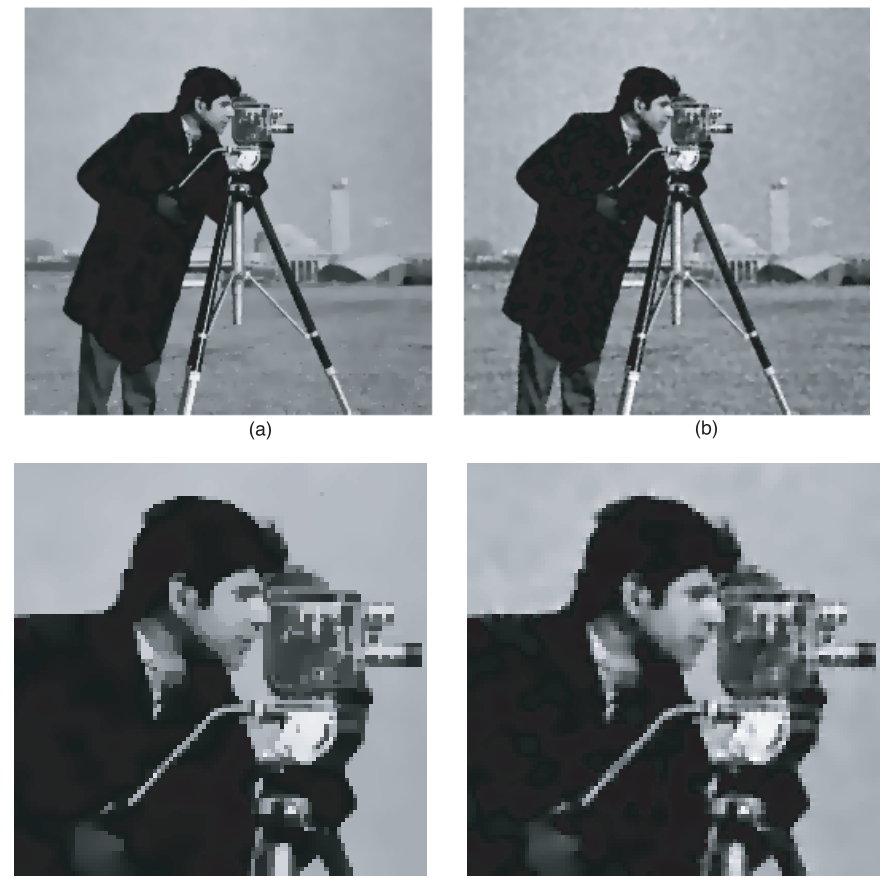

Fig. 7 Comparing the perceptual quality of the results obtained by proposed and PeronaMalik filters for noisy image of Cameraman shown in Fig. 4. The images labeled (a) is the result of the Perona-Malik filter and (b) is the result obtained by the proposed filter. A magnified region of each denoised image is shown in the second row.

become zero and diffusion process is ceased. However, the FOM of the results for both of filters are comparable (i.e., 0.9209 and 0.9260 for the result of PeronaMalik and the proposed filter respectively) owing to the optimality of the choice of $\chi$ in the proposed method.

\section{Conclusion}

A self-governing filter for noise removal based on using the fourth-order PDE has been proposed. A theoretical background of the fourth-order denoising methods has been reviewed with highlighting the major challenges of parameters estimation and slow convergence rate of these techniques. To resolve these draw- 
103 A Self-governing Fourth-order Nonlinear Diffusion Filter

backs, in the proposed method, the diffusion coefficient is calculated by a diffusivity function as a function of modulus of the gradient of the evolved image while, in the existing fourth-order PDE-based filters, the diffusivity function is a function of the absolute value of Laplacian of the image. The simulation results show that the proposed filter can provide a high SNR with a good edge preservation measured by FOM and a tangible improvement of perceptual quality of the denoised images in comparison with those of the other techniques. The fast convergence obtained by the proposed filter pave the road toward its utilization in real time applications.

\section{References}

1) Perona, P. and Malik, J.: Scale-space and edge detection using anisotropic diffusion, IEEE Transactions on Pattern Analysis and Machine Intelligence, Vol.12, No.7, pp.629-639 (1990).

2) Catte, F., et al.: Image selective smoothing and edge detection by nonlinear diffusion, SIAM J. Numer. Anal., Vol.29, No.1, pp.182-193 (1992).

3) Black, M.J., et al.: Robust anisotropic diffusion, IEEE Transactions on Image Processing, Vol.7, No.3, pp.421-432 (1998).

4) You, Y.L. and Kaveh, M.: Fourth-order partial differential equations for noise removal, IEEE Transactions on Image Processing, Vol.9, No.10, pp.1723-1730 (2000).

5) Lysaker, M., Lundervold, A. and Tai, X.-C.: Noise removal using fourth-order partial differential equation with applications to medical magnetic resonance images in space and time, IEEE Tran. on Image Processing, Vol.12, No.12, pp.1579-1590 (2003).

6) Rajan, J., Kannan, K. and Kaimal, M.R.: An Improved hybrid model for molecular image denoising, Journal of Mathematical Imaging and Vision, Vol.31, pp.73-79 (2008).

7) Fang Li, et al.: Image restoration combining a total variational filter and a fourthorder filter, Journal of Visual Communication and Image Representation, Vol.18, pp.322-330 (2007)

8) You, Y.-L., et al.: Behavioral analysis of anisotropic diffusion in image processing, IEEE Trans. Image Processing, Vol.5, pp.1539-1553 (1996).

9) Hamza, A.B., et al.: Removing noise and preserving details with relaxed median filters, Journal of Mathematical Imaging and Vision, Vol.11, No.2, pp.161-177 (1999).

10) Rudin, L., Osher, S. and Fatemi, E.: Nonlinear Total Variation based noise removal algorithms, Physica D, Vol.60, pp.259-268 (1992).

11) Chan T., Marquina A. and Mulet R.: High Order Total Variation-based Image Restoration, SIAM J. on Scientific Computing, Vol.22, No.2, pp.503-516 (2000).

12) Fang, L., et al.: Image restoration combining a total variational filter and a fourthorder filter, Journal of Visual Communication and Image Representation, Vol.18, No.4, pp.322-330 (2007).

13) Nixon, M. and Aguado, A.: Feature Extraction and Image Processing, Oxford, Newnes (2002).

14) Guido, G., et al.: Nonlinear anisotropic filtering of MRI data, IEEE Transactions on Medical Imaging, Vol.11, No.2, pp.221-232 (1992).

15) Canny, J.F.: A computational approach to edge detection, IEEE Transactions on Pattern Analysis and Machine Intelligence, Vol.8, No.6, pp.679-698 (1986).

16) Stoer, J. and Bulirsch, R.: Introduction to Numerical Analysis, Texts in Applied Mathematics, Vol.12, Springer-Verlag, New York (2002).

17) Mazarek, P. and Navara, M.: Selection of Optimal Stopping Time for Nonlinear Diffusion Filtering, International Journal of Computer Vision, Vol.52, No.2/3, pp.189-203 (2003).

18) Pratt, W.K.: Digital Image Processing, New York, Wiley (1977).

(Received April 7, 2009)

(Accepted December 3, 2009)

(Released March 11, 2010)

(Communicated by Toshikazu Wada)

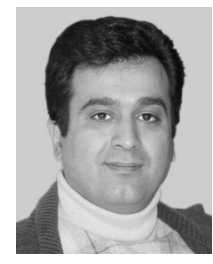

Mohammad Reza Hajiaboli was born in 1972. He received his B.E. degree from Iran University of Science and Technology (Tehran, Iran) and M.Eng. degree from Concordia University (Montreal, Canada), where he was working on CMOS image sensors and their performance at a low illumination condition. He is currently a Ph.D. Candidate in Concordia University, faculty of engineering and computer science. His research focuses on computational imaging and edge preservation smoothing. He is a Student Member of IEEE. 